\title{
Strongly enlarged topological regime and enhanced superconducting gap in nanowires coupled to Ising superconductors
}

\author{
Yingming Xie, Benjamin T. Zhou, ${ }^{*}$ T. K. Ng, and K. T. Law ${ }^{\dagger}$ \\ Department of Physics, Hong Kong University of Science and Technology, Clear Water Bay, 999077 Hong Kong, China
}

(Received 2 September 2019; revised manuscript received 9 December 2019; published 8 January 2020)

\begin{abstract}
An external magnetic field is needed to drive a nanowire in proximity to an $s$-wave superconductor into a topological regime which supports Majorana end states. However, magnetic field generally suppresses the proximity superconducting gap induced on the nanowire. In recent experiments using InSb nanowires coupled to conventional superconductors such as $\mathrm{Al}$ and $\mathrm{NbTiN}$, the induced proximity gap vanishes at magnetic fields $B \sim 1 \mathrm{~T}$. This results in a small superconducting gap on the wire and a narrow topological regime which is proportional to the strength of the magnetic field. In this work, we show that by placing nanowires in proximity to recently discovered Ising superconductors such as the atomically thin transition-metal dichalcogenide $\mathrm{NbSe}_{2}$, the topological superconducting gap on the wire can be maintained at a large magnetic field as strong as $B \sim 10 \mathrm{~T}$. This robust topological superconducting gap is induced by the unique equal-spin triplet Cooper pairs of the parent Ising superconductor. The strong magnetic field allows a topological regime ten times larger than those in InSb wires coupled to conventional superconductors. Our work establishes a realistic platform for building robust Majorana-based qubits.
\end{abstract}

DOI: 10.1103/PhysRevResearch.2.013026

\section{INTRODUCTION}

Majorana zero modes (MZMs) are non-Abelian particles [1] which can serve as potential building blocks of topological quantum computers [2]. It was first shown that MZMs can form at the vortex cores of two-dimensional $p$-wave superconductors [3]. Kitaev later pointed out that this zero-energy mode appears at the ends of a $1 \mathrm{D}$ spinless $p$-wave superconducting wire [4]. Despite the lack of $p$-wave superconductors in nature, effective $p$-wave pairing can be engineered in semiconductors in proximity to conventional $s$-wave superconductors [5-11]. In particular, promising signatures of MZMs have been demonstrated experimentally in semiconducting $\mathrm{InSb}$ nanowires coupled to conventional superconductors upon application of external magnetic fields [12-15]. Recent experimental observation of the predicted quantized zero-bias peak [16] has provided strong evidence for the existence of MZMs and motivated active ongoing efforts toward their applications in topological quantum computations $[17,18]$.

To realize a practical topological quantum computer, a robust topological superconducting gap is essential for the integrity of Majorana-based qubits. However, achieving a hard-gap nanowire has so far been experimentally challenging $[14,16,19]$. One important reason is the dual character of

\footnotetext{
*Corresponding author: bentzhou@ust.hk

†Corresponding author: phlaw@ust.hk
}

Published by the American Physical Society under the terms of the Creative Commons Attribution 4.0 International license. Further distribution of this work must maintain attribution to the author(s) and the published article's title, journal citation, and DOI. magnetic fields in creating topological superconductivity: on one hand, an external magnetic field is needed to create a single species of fermions such that the system becomes an effective spinless Kitaev chain when pairing is introduced; on the other hand, the induced topological gap by usual singlet Cooper pairs gets easily suppressed upon increasing applied magnetic fields $[14,16,19]$. This puts a stringent constraint on the tunable topological regime of the nanowires, thus making the Majorana-based qubits susceptible to applied magnetic fields. A feasible way to engineer a robust topological gap against external fields is clearly desirable.

Recently, Ising superconductors with remarkably high inplane upper critical fields $B_{c 2} \sim 30-50 \mathrm{~T}$ have been discovered in several transition-metal dichalcogenides (TMDs) such as gated $\mathrm{MoS}_{2}$ thin films [20,21] and atomically thin $\mathrm{NbSe}_{2}$ [22]. The strong enhancement of in-plane $B_{c 2}$ was explained to arise from the special Ising spin-orbit coupling (SOC) of TMD materials, which pins electron spins at opposite momentum to opposite out-of-plane directions. Interestingly, with $s$-wave pairing potential only, the Ising SOC generates equal-spin triplet Cooper pairs in Ising superconductors, with their spins pointing to in-plane directions [23]. Under applied in-plane magnetic fields, these equal-spin Cooper pairs align their spin magnetic moments along the direction of applied in-plane fields, which lowers the free energy of Ising superconductors under in-plane fields and gives rise to strongly enhanced inplane $B_{c 2}$.

Importantly, these equal-spin Cooper pairs can tunnel into nanowires under in-plane magnetic fields [23] or magnetic atomic chains $[24,25]$ via proximity effects, which results in a Kitaev chain supporting MZMs. Given the compatibility between these equal-spin Cooper pairs and in-plane spin magnetization, a natural question follows whether the topological 
gap in nanowires created by these Cooper pairs can stay robust against strong in-plane Zeeman fields.

In this work, we show that the answer is positive: the proximity-induced topological gap by the equal-spin Cooper pairs in Ising superconductors is almost insensitive to in-plane Zeeman fields. This is in sharp contrast to the effective $p$ wave pairing created by $s$-wave superconductors, which can be easily suppressed upon application of external magnetic fields.

In particular, by considering a realistic heterostructure formed by InSb nanowires and superconducting monolayer $\mathrm{NbSe}_{2}$, we demonstrate that the topological superconducting gap induced by $\mathrm{NbSe}_{2}$ can persist under applied fields as strong as $B \sim 10 \mathrm{~T}$, which far exceeds the critical field strengths $\left(B_{c} \sim 1 \mathrm{~T}\right)$ in previous experiments using superconducting NbTiN [12]. Importantly, the large external magnetic field creates a large Zeeman gap between the spin-polarized subbands of the InSb wire such that the topological regime, which is proportional to the Zeeman gap, is strongly enlarged. The large superconducting gap and the large topological regime provide a promising platform for realizing Majorana fermions in nanowire/Ising superconductor heterostructures.

In the following sections, we first discuss the important role of equal-spin Cooper pairs in enhancing the in-plane $B_{c 2}$ in Ising superconductors. We explain how these equal-spin Cooper pairs can couple to in-plane external magnetic fields and make the superconducting state energetically favorable at in-plane fields far exceeding conventional Pauli limits. Second, by analytically projecting the contribution of an Ising superconductor onto proximity-coupled nanowires, we show that the equal-spin pairing induced by Ising superconductors is insensitive to in-plane Zeeman fields. Finally, using a realistic tight-binding model, we study the magnetic field dependence of the topological superconducting gap in quasi-1D InSb nanowires placed next to a superconducting monolayer $\mathrm{NbSe}_{2}$. We thus establish a realistic platform based on Ising superconductors for building robust Majorana-based qubits.

\section{ENHANCEMENT OF IN-PLANE $B_{c 2}$ DUE TO EQUAL-SPIN COOPER PAIRS}

In this section, we explain the role of equal-spin Cooper pairs in enhancing in-plane $B_{c 2}$ in Ising superconductors. As we are about to show, these in-plane equal-spin Cooper pairs reconcile the competition between superconductivity and inplane spin magnetization, which creates a robust superconducting order in Ising superconductors against in-plane fields.

In the following discussions, we ignore the orbital-pairbreaking effects of in-plane fields. This simplification is valid given that the recently found Ising superconductors such as $2 \mathrm{H}-\mathrm{NbSe}_{2}$ [22] or $2 \mathrm{H}-\mathrm{MoS}_{2}[20,21]$ have atomic-scale thickness far smaller than the superconducting coherence length.

In general, the normal state of an Ising superconductor can be described by the following two-band model Hamiltonian:

$$
H_{0}=\sum_{\boldsymbol{k}, s_{1}, s_{2}} c_{\boldsymbol{k} s_{1}}^{\dagger}\left\{\xi(\boldsymbol{k}) \delta_{s_{1}, s_{2}}+[\boldsymbol{g}(\boldsymbol{k}) \cdot \boldsymbol{\sigma}]_{s_{1}, s_{2}}\right\} c_{\boldsymbol{k} s_{2}},
$$

where $\xi(\boldsymbol{k})=\frac{\hbar^{2} \boldsymbol{k}^{2}}{2 m}-\mu$ is the kinetic-energy term, $\boldsymbol{\sigma}$ operates on the spin space, and the $\boldsymbol{g}(\boldsymbol{k})$ term represents generic noncentrosymmetric spin-orbit coupling (SOC) terms, with $\boldsymbol{g}(\boldsymbol{k})=-\boldsymbol{g}(-\boldsymbol{k})$ imposed by time-reversal symmetry. For intrinsic Ising superconductors such as monolayer $\mathrm{NbSe}_{2}$, the $D_{3 h}$ point group symmetry of $2 H$-TMDs dictates that the SOC terms can only pin electron spins to the out-of-plane directions, i.e., $\boldsymbol{g}(\boldsymbol{k})=(0,0, \beta(\boldsymbol{k}))$, thus referred to as the Ising SOC. The effect of Zeeman fields is included by rewriting $\boldsymbol{g}(\boldsymbol{k})$ as $\boldsymbol{g}(\boldsymbol{k}, \boldsymbol{V})=\boldsymbol{g}(\boldsymbol{k})+\boldsymbol{V}$, where $\boldsymbol{V}$ is given by the product between the Bohr magneton $u_{B}$ and the applied magnetic field $\boldsymbol{B}$. Because of the presence of Ising SOC, the normal state spectrum exhibits a band splitting given by $\xi_{ \pm}(\boldsymbol{k})=\xi(\boldsymbol{k}) \pm$ $|g(k)|$.

For the superconducting state, we assume the dominant pairing channel is the on-site attractive interaction, and the mean-field pairing potential is simply given by

$$
H_{\text {pair }}=\sum_{\boldsymbol{k}} \sum_{s_{1}, s_{2}} \Delta\left(i \sigma_{y}\right)_{s_{1}, s_{2}} c_{\boldsymbol{k} s_{1}}^{\dagger} c_{-\boldsymbol{k} s_{2}}^{\dagger}+\text { H.c., }
$$

where $\Delta$ is the $s$-wave order parameter. As pointed out by previous works [26], the spin structure of Cooper pairs in a superconductor with SOC can be conveniently described by the pairing correlation defined as

$$
F_{\alpha \beta}\left(\boldsymbol{k}, \tau_{1} ; \boldsymbol{k}^{\prime}, \tau_{2}\right)=\left\langle T_{\tau} c_{\boldsymbol{k}, \alpha}\left(\tau_{1}\right) c_{-\boldsymbol{k}^{\prime}, \beta}\left(\tau_{2}\right)\right\rangle .
$$

In the Matsubara frequency space, the pairing correlation $F$ can be written as a compact matrix form [26]:

$$
F\left(\boldsymbol{k}, i \omega_{n}\right)=\left[F_{s}\left(\boldsymbol{k}, i \omega_{n}\right)+\boldsymbol{F}_{t}\left(\boldsymbol{k}, i \omega_{n}\right) \cdot \boldsymbol{\sigma}\right] \Delta i \sigma_{y},
$$

where $F_{s}$ and $\boldsymbol{F}_{t}$ parametrize the singlet and triplet correlation functions, respectively. By solving the Gor'kov equations (see Appendix A for details), $F_{s}$ and $\boldsymbol{F}_{t}$ can be obtained as

$$
\begin{gathered}
F_{s}\left(\boldsymbol{k}, i \omega_{n}\right)=\frac{1}{2}\left[\frac{1}{\varphi_{+}\left(\boldsymbol{k}, \Delta, \omega_{n}\right)}+\frac{1}{\varphi_{-}\left(\boldsymbol{k}, \Delta, \omega_{n}\right)}\right], \\
\boldsymbol{F}_{t}\left(\boldsymbol{k}, i \omega_{n}\right)=\frac{1}{2}\left[\frac{1}{\varphi_{+}\left(\boldsymbol{k}, \Delta, \omega_{n}\right)}-\frac{1}{\varphi_{-}\left(\boldsymbol{k}, \Delta, \omega_{n}\right)}\right] \hat{\boldsymbol{g}}(\boldsymbol{k}),
\end{gathered}
$$

with the specific forms of $\varphi_{ \pm}$given in Appendix A. Notably, because of the presence of SOC, there is a nonzero triplet pairing correlation even though the mean-field potential is $s$ wave $[23,27,28]$. In particular, the triplet pairing correlation is parametrized by a vector-valued function $\boldsymbol{F}_{t}$. For any fixed $\boldsymbol{k}$, $\boldsymbol{F}_{t}$ is parallel to the SOC vector $\boldsymbol{g}$ according to Eq. (6).

In the specific case of Ising superconductors, the unit SOC vector $\hat{\boldsymbol{g}}=\hat{z}$, which implies that the triplet correlation $\boldsymbol{F}_{t}$ has a nonzero $z$ component only. Under the basis of out-of-plane spin states (the out-of-plane $z$ axis defined as the spin quantization axis), Eq. (6) suggests that the triplet Cooper pairs are formed by electrons of opposite out-of-plane spins; i.e., the spinor wave function is given by $|\uparrow \downarrow\rangle+|\downarrow \uparrow\rangle$. Interestingly, by a straightforward change of basis from out-of-plane to in-plane spins, these triplet Cooper pairs have their spinor part given by equal-spin configurations. Without loss of generality, by defining the $x$ axis as the spin quantization axis, the triplet state becomes $|\uparrow \downarrow\rangle+|\downarrow \uparrow\rangle \equiv|\rightarrow \rightarrow\rangle-|\leftarrow \leftarrow\rangle$. Thus, the spinor wave function of these triplet Cooper pairs is an equal superposition of spinor states with opposite in-plane spin magnetic moments. Under applied in-plane fields $B_{x}$, these in-plane equal-spin Cooper pairs can align their spin magnetic moments along the magnetic field direction. This 

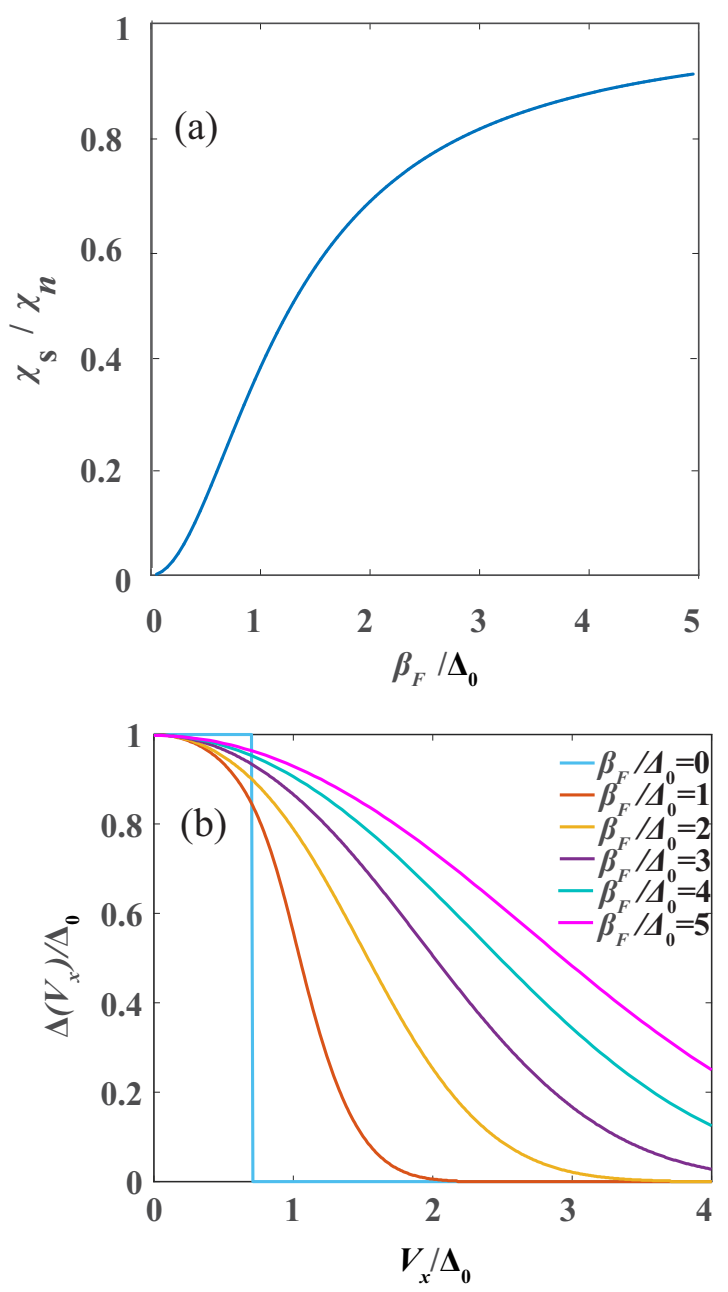

FIG. 1. (a) The superconducting spin susceptibility $\chi_{s}$ as a function of Ising SOC strength $\beta_{F}$ at the Fermi energy. Without Ising $\operatorname{SOC}\left(\beta_{F}=0\right), \chi_{s}=0$. As Ising SOC is turned on, spin triplet Cooper pairs start to form in Ising superconductors and give rise to a nonzero $\chi_{s}$. (b) Superconducting gap $\Delta$ obtained self-consistently [Eq. (B4)] at zero temperature as a function of Zeeman energy $V_{x}=\mu_{B} B_{x}$ under different Ising SOC strengths. When $\beta_{F}=0$, superconductivity is destroyed at the usual Pauli limit: $B_{p}=\Delta_{0} /\left(\sqrt{2} \mu_{B}\right)$. When the strength of Ising SOC is finite, the upper critical field is enhanced, which exceeds $B_{p}$ by several times.

endows the Ising superconductor with a finite spin susceptibility as shown in Fig. 1(a). As a result, an Ising superconductor gains magnetic energy under in-plane magnetic fields, with its superconducting free energy kept lowering as the field strength increases. This leads to an enhanced in-plane $B_{c 2}$.

To demonstrate the enhancement of $B_{c 2}$ due to Ising SOC, we solve the superconducting gap self-consistently as a function of in-plane magnetic field. Without loss of generality, we assume $\boldsymbol{V}=V_{x} \hat{x}$, and the self-consistent gap equation is given by

$$
\Delta i \sigma_{y}=T \sum_{\boldsymbol{k}, n} V_{0} F_{s}\left(\boldsymbol{k}, V_{x}, i \omega_{n}\right) \Delta i \sigma_{y},
$$

where $V_{0}$ is the interaction strength and $F_{s}\left(\boldsymbol{k}, V_{x}, i \omega_{n}\right)$ is the pairing correlation including the magnetic field. Details of

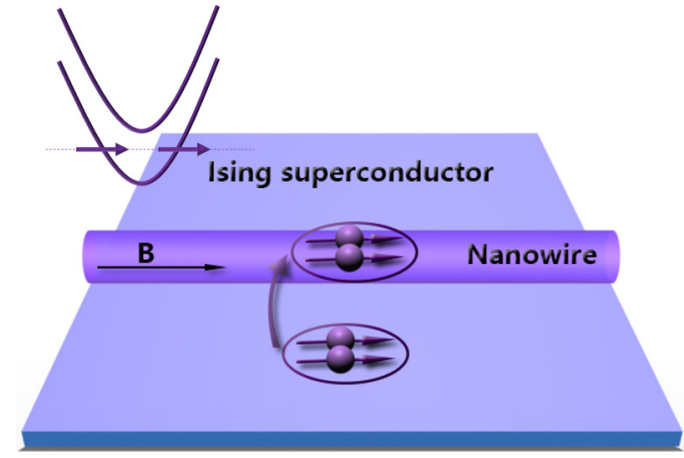

FIG. 2. Schematic of a nanowire in proximity to an Ising superconductor. $B$ is the in-plane magnetic field required to induce sizable spin splitting such that a single band is filled at the Fermi energy. The nanowire acquires a proximity gap from the equal-spin Cooper pairs in Ising superconductors and becomes topologically equivalent to a Kitaev chain.

the self-consistent gap equations and spin susceptibilities are presented in Appendices A and B.

In the zero-temperature limit, the superconducting gaps as a function of $V_{x}=\mu_{B} B_{x}$ with different Ising SOC strengths are shown in Fig. 1(b). Without Ising SOC, superconductivity is destroyed by the paramagnetic effect at the Pauli limit $B_{p} \approx \Delta_{0} / \sqrt{2} \mu_{B}$ [light blue curve in Fig. 1(b)]. The superconductor undergoes a first-order phase transition with its order parameter vanishing abruptly to zero. In contrast, in the presence of Ising SOC, the upper critical magnetic fields are strongly enhanced to several times the usual Pauli limit. Moreover, the superconducting gap decreases gradually to zero as $V_{x}$ increases, signifying a continuous phase transition at $B_{c 2}$. This continuous superconductor-normal phase transition has recently been observed experimentally in atomically thin $\mathrm{NbSe}_{2}$ [29], which confirms the robust superconducting order of Ising superconductors against in-plane magnetic fields.

\section{ROBUST TOPOLOGICAL SUPERCONDUCTIVITY IN NANOWIRES COUPLED TO ISING SUPERCONDUCTORS}

In this section, we study in detail the superconducting proximity effects in semiconducting nanowires (NWs), such as InSb NWs, placed on top of an Ising superconductor. In particular, we demonstrate how a robust topological gap can be created in InSb NWs using the equal-spin Cooper pairs in Ising superconductors.

In heterostructures formed by InSb NWs and conventional superconductors, the proximity-induced gap originates from the parent $s$-wave superconducting gap, which can be easily suppressed under strong magnetic fields [6,7]. However, the situation can be very different using an Ising superconductor. First, as we demonstrated in the previous section, the parent superconducting gap of Ising superconductors is much less sensitive to external in-plane fields. Second, the special equalspin Cooper pairs from Ising superconductors, which are compatible with in-plane fields, can tunnel into InSb nanowires via proximity effect as depicted in Fig. 2. As expected, they can create a robust proximity gap which stays robust at much higher in-plane fields. 


\section{A. Strictly one-dimensional nanowire on a generic Ising superconductor}

To study the proximity-induced superconducting pairing, we first consider a strictly one-dimensional InSb nanowire placed on a generic Ising superconductor described by the simple two-band model presented in the previous section. The Hamiltonian of Ising superconductors in the Nambu basis $\left(\psi_{\boldsymbol{k} \uparrow}^{(s)}, \psi_{\boldsymbol{k} \downarrow}^{(s)}, \psi_{-\boldsymbol{k} \uparrow}^{(s)}, \psi_{-\boldsymbol{k} \downarrow}^{\dagger(s)}\right)^{T}$ is

$$
H_{s}=\sum_{\boldsymbol{k}} \psi^{\dagger(s)}(\boldsymbol{k}) \mathcal{H}_{\mathrm{BdG}}(\boldsymbol{k}) \psi^{(s)}(\boldsymbol{k}),
$$

where

$$
\mathcal{H}_{\mathrm{BdG}}(\boldsymbol{k})=\left(\begin{array}{cc}
H_{0}(\boldsymbol{k}) & \Delta i \sigma_{y} \\
\left(\Delta i \sigma_{y}\right)^{\dagger} & -H_{0}^{T}(-\boldsymbol{k})
\end{array}\right) .
$$

In the basis $\left[c_{\uparrow}^{(w)}(x), c_{\downarrow}^{(w)}(x), c_{\uparrow}^{(w) \dagger}(x), c_{\downarrow}^{(w) \dagger}(x)\right]^{T}$, the nanowire under in-plane magnetic field can be described by

$$
\begin{aligned}
H_{w}= & \int d x c^{(w) \dagger}(x)\left[\left(\frac{\hbar^{2} \hat{k}_{x}^{2}}{2 m}-\mu_{w}\right) \tau_{z}+\alpha_{R} \hat{k}_{x} \sigma_{y} \tau_{z}\right. \\
& \left.+V_{x} \sigma_{x} \tau_{z}\right] c^{(w)}(x) .
\end{aligned}
$$

The tunneling Hamiltonian is

$$
H_{s-w}=\int d x \psi^{(s) \dagger}(x) \mathcal{T} c^{(w)}(x)+\text { H.c. },
$$

where $\mathcal{T}=\Gamma_{c} \tau_{z}, \Gamma_{c}$ is the coupling strength, $\tau_{z}$ operates on particle-hole space, $\mu_{w}$ is the chemical potential of the nanowire, and $\alpha_{R}$ is the Rashba SOC strength.

The contribution from the Ising superconductor is included as a self-energy term:

$$
\begin{aligned}
\Sigma\left(x, x^{\prime} ; i \omega\right) & =\int \frac{d k_{x} d \boldsymbol{k}_{\perp}}{(2 \pi)^{2}} \mathcal{T}^{\dagger} \mathcal{G}(\boldsymbol{k}, i \omega) \mathcal{T} e^{-i k_{x}\left(x-x^{\prime}\right)} \\
& =\int \frac{d k_{x}}{2 \pi} \Sigma\left(k_{x} ; i \omega\right) e^{-i k_{x}\left(x-x^{\prime}\right)},
\end{aligned}
$$

where $\quad \Sigma\left(k_{x} ; i \omega\right)=\mathcal{T}^{\dagger} \mathcal{G}^{\mathrm{Sur}}(\boldsymbol{k}, i \omega) \mathcal{T}, \quad \mathcal{G}^{\mathrm{Sur}}(\boldsymbol{k}, i \omega)=$ $\int \frac{d \boldsymbol{k}_{\perp}}{2 \pi} \mathcal{G}(\boldsymbol{k}, i \omega)$. As shown in Appendix $\mathrm{C}$, including $\Sigma$, the low-energy effective Hamiltonian of the nanowires is written as

$$
\hat{H}_{\text {eff }}\left(k_{x}\right)=\left(\begin{array}{cc}
\tilde{h}\left(k_{x}\right) & \tilde{\Delta}\left(k_{x}\right) \\
\tilde{\Delta}\left(k_{x}\right)^{\dagger} & -\tilde{h}^{T}\left(-k_{x}\right)
\end{array}\right),
$$

where $\tilde{\Delta}\left(k_{x}\right)=\left[\psi\left(k_{x}\right)+\boldsymbol{d}\left(k_{x}\right) \cdot \boldsymbol{\sigma}\right] i \sigma_{y}$ denotes the proximityinduced pairing matrix. Details of $\tilde{h}\left(k_{x}\right), \psi\left(k_{x}\right), \boldsymbol{d}\left(k_{x}\right)$ are presented in Appendix C.

Now we study the components in $\tilde{\Delta}\left(k_{x}\right)$ which can give rise to topological superconductivity. Consider the case in which the magnetic field is parallel to the nanowire, with its strength large enough that only a single band in the wire is occupied at the Fermi energy. To see the nontrivial induced pairing in the lowest band, we follow Ref. [30] to rewrite the Hamiltonian using the band basis, and the resulting effective $p$-wave pairing $\Delta^{(p)}$ is given by

$$
\Delta^{(p)}=\Delta_{s, \alpha}^{(p)}+\Delta_{s, \beta}^{(p)}+\Delta_{t}^{(p)},
$$

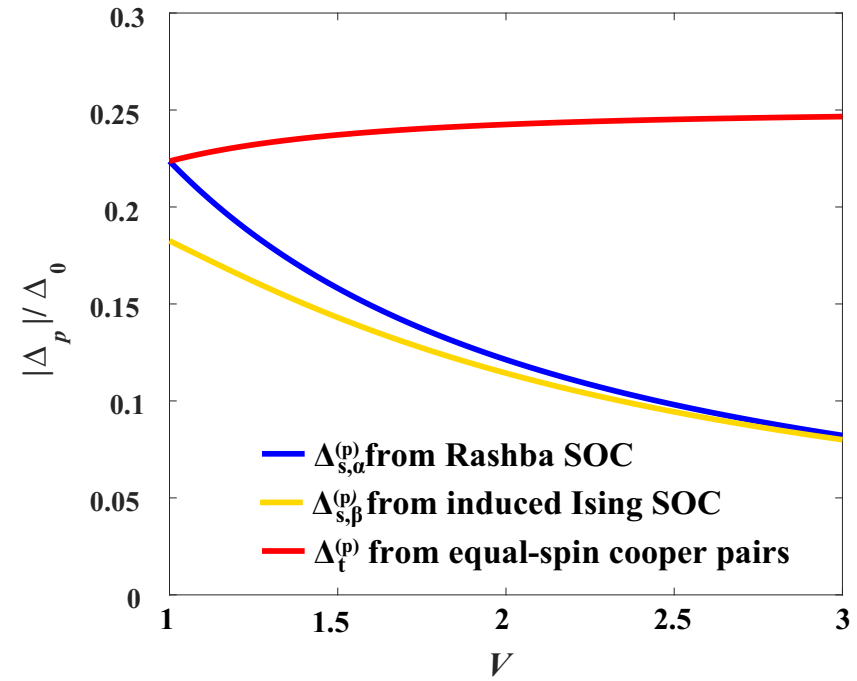

FIG. 3. Different $p$-wave pairing gaps as a function of $V$, where $V=\tilde{\mathcal{Z}}_{0}^{-1} V_{x} / \Delta_{0}$ is a dimensionless parameter that characterizes the renormalized Zeeman effect. The blue and yellow curves represent $p$-wave pairing gap $\Delta_{s}^{(p)}$ induced by spin-singlet Cooper pairs and Rashba and induced Ising SOCs. Clearly, both of the induced gaps $\Delta_{s}^{(p)}$ are suppressed upon increasing $V$. In contrast, the $p$-wave pairing gap $\Delta_{t}^{(p)}$ induced by equal-spin triplet Cooper pairs from Ising superconductors is insensitive to $V$. Here, we set the parameters to be $\alpha=0.5, \beta=0.5, d_{z}\left(k_{F}\right) / \Delta_{0}=0.5$.

$$
\begin{aligned}
\Delta_{s, \alpha}^{(p)} & \approx \frac{\alpha}{2 \sqrt{V^{2}+\alpha^{2}}} \Delta_{0}, \\
\Delta_{s, \beta}^{(p)} & \approx \frac{V \beta}{2 \sqrt{V^{2}+\alpha^{2}+\beta^{2}} \sqrt{V^{2}+\alpha^{2}}} \Delta_{0}, \\
\Delta_{t}^{(p)} & \approx-\frac{V}{2 \sqrt{V^{2}+\alpha^{2}}} d_{z}\left(k_{x}\right) .
\end{aligned}
$$

Notably, there are three $p$-wave pairing terms in Eq. (15) arising from different physical origins. We denote the effective $p$-wave pairing due to singlet Cooper pairs as $\Delta_{s}^{(p)}$, and the pairing due to equal-spin triplet Cooper pairs as $\Delta_{t}^{(p)}$.

The first $\Delta_{s, \alpha}^{(p)}$ term is the well studied effective $p$-wave pairing induced by Rashba SOC, the Zeeman effect, and the proximity-induced $s$-wave pairing potential (blue curve in Fig. 3), which is responsible for creating topological superconductivity in conventional InSb nanowires with Rashba SOC. Here, $\alpha=\tilde{\mathcal{Z}}_{0}^{-1} \alpha_{R} k_{x} / \Delta_{0}$ characterizes the strength of Rashba SOC strength in the nanowire, $V=\tilde{\mathcal{Z}}_{0}^{-1} V_{x} / \Delta_{0}$ is the renormalized Zeeman energy, and $\Delta_{0}$ is the induced on-site $s$-wave component in $\psi\left(k_{x}\right)$.

The second $\Delta_{s, \beta}^{(p)}$ term also results from proximity-induced singlet pairing (yellow curve in Fig. 3), where $\beta=\beta_{I}\left(k_{x}\right) / \Delta_{0}$ is the proximity-induced Ising SOC in the nanowire. It arises from a physical mechanism similar to that of the $\Delta_{s, \alpha}^{(p)}$ term, except that the Ising SOC is induced in the nanowires from the background Ising superconductor. Notably, Eq. (15) indicates that both $\Delta_{s, \alpha}^{(p)}$ and $\Delta_{s, \beta}^{(p)}$ get strongly suppressed as the Zeeman effect dominates: $V \gg \alpha, \beta$. 
In contrast, the last $\Delta_{t}^{(p)}$ term in Eq. (15) describes a triplet pairing which originates from the intrinsic equal-spin Cooper pairs in the parent Ising superconductor, with $d_{z}\left(k_{x}\right) \propto k_{x}$ (Appendix C). It has a weak dependence on Zeeman energy $V$ and in the limit $V \gg \alpha, \beta$, we have $\Delta_{t}^{(p)} \approx-d_{z}\left(k_{x}\right) / 2$. Thus, $\Delta_{t}^{(p)}$ stays robust against Zeeman fields.

To make an explicit comparison among all $p$-wave pairing terms above, we calculate the amplitude of each term as a function of Zeeman energy $V$ (Fig. 3). Evidently, both $\Delta_{s, \alpha}^{(p)}$ and $\Delta_{s, \beta}^{(p)}$ from intrinsic Rashba SOC and induced Ising SOC gradually decrease upon increasing $V_{x}$ (blue and yellow curves in Fig. 3), as the magnetic field tends to pin spins to the same directions and competes with the opposite spin-singlet pairing. In contrast, since $\Delta_{t}^{(p)}$ arises from equal-spin Cooper pairs with spins pointing to in-plane directions, it is compatible with in-plane fields and its amplitude remains almost unaffected by the in-plane field (red curve in Fig. 3).

\section{B. Quasi-one-dimensional nanowires on superconducting atomically thin $\mathrm{NbSe}_{2}$}

In the previous subsection, we use a simplified strictly $1 \mathrm{D}$ model for InSb nanowires and a simple two-band model for Ising superconductors to illustrate the robust topological superconducting gap induced by equal-spin Cooper pairs. Here, we consider a realistic heterostructure formed by quasi-onedimensional nanowires and a specific Ising superconductor, monolayer $2 \mathrm{H}-\mathrm{NbSe}_{2}$ [31], to study the proximity-induced gap numerically. The setup considered here is the same as in Fig. 2.

The realistic tight-binding model for monolayer $2 \mathrm{H}-\mathrm{NbSe}_{2}$ $[31]$ is

$$
\begin{aligned}
H_{\mathrm{TMD}}= & \sum_{\boldsymbol{R}, \alpha}\left(\epsilon_{\alpha}-\mu\right) \psi_{\alpha, s}^{\dagger}(\boldsymbol{R}) \psi_{\alpha, s}(\boldsymbol{R}) \\
& +\sum_{\boldsymbol{R}, \boldsymbol{d}, s, \alpha, \beta} t_{\alpha \beta}(\boldsymbol{d}) \psi_{\alpha, s}^{\dagger}(\boldsymbol{R}) \psi_{\beta, s}(\boldsymbol{R}+\boldsymbol{d})+\text { H.c. } \\
& +\sum_{\boldsymbol{R}, s, s^{\prime}, \alpha, \beta} \beta_{\mathrm{so}} \psi_{\alpha, s}^{\dagger}(\boldsymbol{R})\left(\sigma_{z}\right)_{s s^{\prime}}\left(L_{z}\right)_{\alpha \beta} \psi_{\beta, s^{\prime}}(\boldsymbol{R}) \\
& +\sum_{\boldsymbol{R}, s, s^{\prime}, \alpha} \Delta \psi_{\alpha, s}^{\dagger}(\boldsymbol{R})\left(i \sigma_{y}\right)_{s s^{\prime}} \psi_{\alpha, s^{\prime}}^{\dagger}(\boldsymbol{R})+\text { H.c. }
\end{aligned}
$$

The tight-binding Hamiltonian for the semiconductor nanowire is

$$
\begin{aligned}
H_{w}= & \sum_{\boldsymbol{R}, s, s^{\prime}} c_{s}^{\dagger}(\boldsymbol{R})\left[\left(4 t_{w}-\mu_{w}\right) \delta_{s s^{\prime}}+V_{x}\left(\sigma_{x}\right)_{s s^{\prime}}\right] c_{s^{\prime}}(\boldsymbol{R}) \\
& +\sum_{\boldsymbol{R}, \boldsymbol{d}, s}-t_{w} c_{s}^{\dagger}(\boldsymbol{R}) c_{s}(\boldsymbol{R}+\boldsymbol{d})+\text { H.c. } \\
& +\sum_{\boldsymbol{R}, \boldsymbol{d}, s, s^{\prime}} \frac{i}{2} \alpha_{R} c_{s}^{\dagger}(\boldsymbol{R}) \hat{\boldsymbol{e}}_{z} \cdot\left(\boldsymbol{\sigma}_{s s^{\prime}} \times \boldsymbol{d}\right) c_{s^{\prime}}(\boldsymbol{R}+\boldsymbol{d})+\text { H.c. }
\end{aligned}
$$

The tunneling Hamiltonian is

$$
H_{c}=\sum_{\boldsymbol{R} \in \text { wire }, \alpha, s} \Gamma_{c} \psi_{\alpha, s}^{\dagger}(\boldsymbol{R}) c_{s}(\boldsymbol{R})+\text { H.c. }
$$

TABLE I. Parameters for tight-binding model estimated from Refs. [12,22]. Parameters for $2 \mathrm{H}-\mathrm{NbSe}_{2}$ are adapted from $a b$ initiobased calculations in Ref. [32].

\begin{tabular}{lcc}
\hline \hline Parameter & Symbol & Value \\
\hline Lattice constant of wire & $a$ & $6 \AA$ \\
Rashba parameter & $\alpha_{R}$ & $0.2 \mathrm{eV} \mathrm{\AA}$ \\
Hopping strength of wire & $t_{w}$ & $250 \mathrm{eV}{ }^{2}$ \\
Magnetic energy & $V_{x}$ & $1.5 \mathrm{meV} / T \times B$ \\
Superconducting gap & $\Delta$ & $0.5 \mathrm{meV}$ \\
Coupling strength & $\Gamma_{c}$ & $120 \Delta$ \\
\hline \hline
\end{tabular}

The total Hamiltonian is

$$
H_{t}=H_{\mathrm{TMD}}+H_{w}+H_{c},
$$

where $\alpha, \beta$ label different orbitals, $s, s^{\prime}$ label the spin, the Pauli matrix $\sigma$ is defined in spin space, $L_{z}$ is the angular momentum operator defined in orbital space, $\epsilon_{\alpha}$ is the on-site energy for orbital $\alpha, \boldsymbol{d}$ is a lattice vector connecting the nearest and next-nearest sites of the TMD or the nearest sites of the nanowire, $t_{\alpha \beta}(\boldsymbol{d}), t_{w}$ are the hopping strength, $\mu, \mu_{w}$ are the chemical potentials of the TMD and nanowire, $\beta_{\text {so }}$ is the strength of Ising SOC, $\alpha_{R}$ is the Rashba SOC parameter, $\Gamma_{c}$ is the coupling strength between the TMD and nanowire, and $\psi_{\alpha, s}(\boldsymbol{R})$ and $c_{s}(\boldsymbol{R})$ are annihilation operators of the TMD and the nanowire.

In order to see how the pairing gap changes with magnetic field, we use realistic parameters of InSb nanowires [12,18] and monolayer $2 \mathrm{H}-\mathrm{NbSe}_{2}[31,32]$ to calculate the local density of states (LDOS) at the end of the wire as a function of $V_{x}$ (Fig. 4). For a straightforward comparison, we model a usual $s$-wave superconductor by setting the Ising SOC to be zero in the $\mathrm{NbSe}_{2}$. Details of these parameters are shown in Table I.

The LDOS at one end of the InSb nanowires coupled to superconducting monolayer $\mathrm{NbSe}_{2}$ is shown in Fig. 4(a). Clearly, upon increasing magnetic field, the bulk excitation gap closes and reopens at $B \approx 0.5 \mathrm{~T}$. The system enters the topologically nontrivial regime at the gap closing point, signified by the appearance of Majorana zero modes. Consistently with Fig. 3, the topological superconducting gap of the InSb wire remains sizable for field strengths up to $B=5 \mathrm{~T}$ according to our numerical calculations in Fig. 4(a). Notably, for magnetic fields larger than $5 \mathrm{~T}$, the wire still remains a topological superconductor as long as the proximity gap is finite. The proximity gap can eventually be destroyed if the parent superconducting gap in $\mathrm{NbSe}_{2}$ is closed by the applied magnetic field. This can indeed happen at a field strength $B \sim$ 10 T corresponding to the conventional Pauli limit, where the parent superconducting $\mathrm{NbSe}_{2}$ becomes a nodal topological superconductor [32]. Therefore, the topological regime of the InSb wire is significantly enlarged with $B$ field ranging from 0.5 to $10 \mathrm{~T}$. The Majorana nature of the lowest-lying zero-energy modes in Fig. 4(a) is further confirmed by the oscillation due to hybridized Majorana modes in a finitely long wire (Appendix D).

On the contrary, as shown in Fig. 4(b), for a conventional parent superconductor with spin-singlet Cooper pairs only, the topological superconducting gap is suppressed quickly. To 

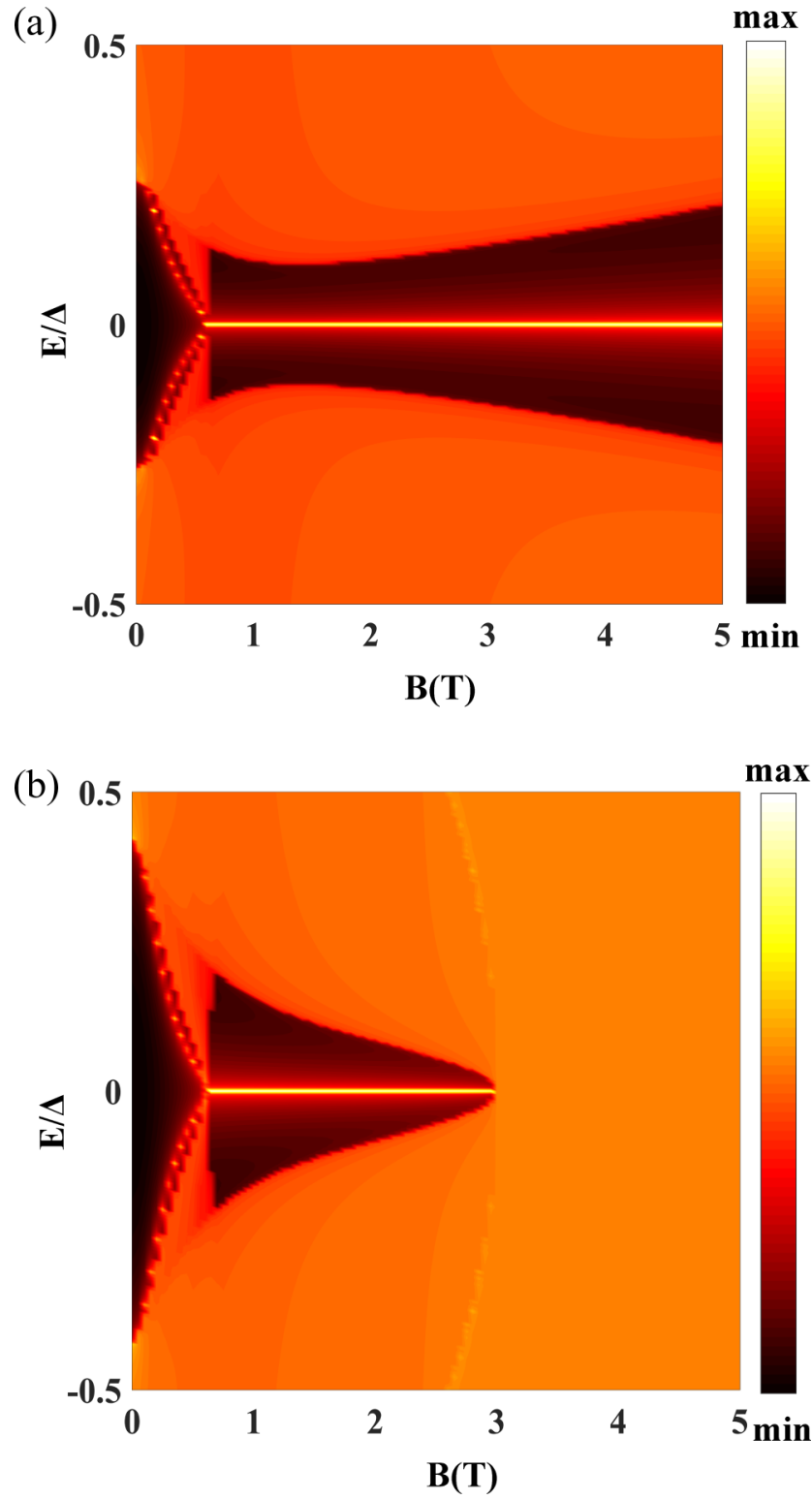

FIG. 4. Comparison between topological superconducting gaps in (a) the InSb nanowire/ $\mathrm{NbSe}_{2}$ heterostructure and (b) the InSb nanowire/conventional superconductor heterostructure. (a) Local density of states at the end of InSb nanowires coupled to atomically thin superconducting $\mathrm{NbSe}_{2}$. The topological gap remains large at in-plane fields of a few teslas, and persists up to conventional Pauli limiting field $B \sim 10 \mathrm{~T}$ [32] of the parent superconductor $\mathrm{NbSe}_{2}$. The value of $\beta_{\text {so }}$ is taken from first-principles calculations in Ref. [32]. (b) Local density states at the end of the wire on top of a conventional superconductor without Ising SOC. The topological gap shows a typical decaying behavior. We set the magnitude of the parent superconducting gap as $\Delta \sqrt{1-\left(B / B_{c}\right)^{2}}$ [33]. The critical field $B_{c}$ is set to be $3 \mathrm{~T}$.

illustrate the differences between the InSb nanowire/ $\mathrm{NbSe}_{2}$ heterostructure and the InSb nanowire/conventional superconductor heterostructures under realistic experimental conditions, in Fig. 4(b) we also considered the orbital-pairbreaking effects, which were found to cause a magnetic field dependence of the parent gap in conventional superconduc- tors: $\Delta(B)=\Delta \sqrt{1-\left(B / B_{c}\right)^{2}}$ with $B_{c} \approx 3 \mathrm{~T}[13,33]$. As we mentioned earlier, the orbital-pair-breaking effect is negligible in atomically thin $\mathrm{NbSe}_{2}$ as its thickness is far smaller than the superconducting coherence length. Thus, we conclude that an Ising superconductor such as atomically thin $\mathrm{NbSe}_{2}$ has an overall advantage in inducing a robust topological gap in $\mathrm{InSb}$ nanowires and a strongly enlarged topological regime under external magnetic fields.

\section{CONCLUSION}

In conclusion, we showed that Ising superconductors such as atomically thin superconducting $\mathrm{NbSe} 2$ can induce a robust topological superconducting gap in InSb nanowires with a strongly enlarged topological regime. We explained that the robust proximity gap originates from the special equal-spin Cooper pairs in Ising superconductors which are compatible with in-plane magnetic fields. This robust topological superconducting gap with a wide topological regime in Majorana nanowires induced by Ising superconductors provides a promising platform for robust Majorana-based qubits.

\section{ACKNOWLEDGMENTS}

The authors thank Noah F. Yuan for helpful discussions. K.T.L. acknowledges the support of the Croucher Foundation, the Dr. Tai-chin Lo Foundation, and HKRGC through Grants No. C6026-16W, No. 16324216, No. 16307117, and No. 16309718.

\section{APPENDIX A: PAIRING CORRELATION FROM THE GOR'KOV EQUATION}

By solving the Gor'kov equation, we obtain

$$
\begin{aligned}
F_{s}\left(\boldsymbol{k}, \boldsymbol{V}, i \omega_{n}\right)= & \frac{1}{2}\left(\frac{1}{\varphi_{+}}+\frac{1}{\varphi_{-}}\right), \\
\boldsymbol{F}_{t}\left(\boldsymbol{k}, \boldsymbol{V}, i \omega_{n}\right)= & \left\{-\left[\xi(\boldsymbol{k})+i \omega_{n}\right] \boldsymbol{g}(\boldsymbol{k}, \boldsymbol{V})+\left[\xi(\boldsymbol{k})-i \omega_{n}\right]\right. \\
& \times \boldsymbol{g}(-\boldsymbol{k}, \boldsymbol{V})-i \boldsymbol{g}(\boldsymbol{k}, \boldsymbol{V}) \times \boldsymbol{g}(-\boldsymbol{k}, \boldsymbol{V})\} /\left(\varphi_{+} \varphi_{-}\right),
\end{aligned}
$$

where $\varphi_{ \pm} \equiv \varphi_{ \pm}\left(\boldsymbol{k}, \boldsymbol{V}, \Delta, \omega_{n}\right)$ are explicitly given by $\varphi_{ \pm}\left(\boldsymbol{k}, \boldsymbol{V}, \Delta, \omega_{n}\right)=\Delta^{2}+\omega_{n}^{2}-|\boldsymbol{V}|^{2}+|\boldsymbol{g}(\boldsymbol{k})|^{2}+\xi^{2}(\boldsymbol{k}) \pm$ $2\left[|\boldsymbol{g}(\boldsymbol{k})|^{2} \xi^{2}(\boldsymbol{k})-|\boldsymbol{V}|^{2} \omega_{n}^{2}+2 i \boldsymbol{g}(\boldsymbol{k}) \cdot \boldsymbol{V} \xi(\boldsymbol{k}) \omega_{n}-\mid \boldsymbol{g}(\boldsymbol{k}) \times\right.$ $\left.\left.\boldsymbol{V}\right|^{2}\right]^{1 / 2}$ and $F\left(\boldsymbol{k}, \boldsymbol{V}, i \omega_{n}\right)$ has been parametrized as spin singlet pairing correlations $F_{s}\left(\boldsymbol{k}, \boldsymbol{V}, i \omega_{n}\right)$ and spin triplet pairing correlations $\boldsymbol{F}_{t}\left(\boldsymbol{k}, \boldsymbol{V}, i \omega_{n}\right)$, where $F\left(\boldsymbol{k}, \boldsymbol{V}, i \omega_{n}\right)=$ $\left[F_{s}\left(\boldsymbol{k}, \boldsymbol{V}, i \omega_{n}\right)+\boldsymbol{F}_{t}\left(\boldsymbol{k}, \boldsymbol{V}, i \omega_{n}\right) \cdot \boldsymbol{\sigma}\right] \Delta i \sigma_{y}$.

\section{APPENDIX B: SELF-CONSISTENT GAP EQUATION FOR ISING SUPERCONDUCTORS}

The self-consistent gap equation for the Ising superconductor under an in-plane magnetic field is given by

$$
\Delta i \sigma_{y}=T \sum_{\boldsymbol{k}, n} V_{0} F_{s}\left(\boldsymbol{k}, V_{x}, i \omega_{n}\right) \Delta i \sigma_{y}
$$


The nontrivial solution $\Delta$ is obtained by solving Eq. (B1) self-consistently. Substituting Eq. (A1), we obtain

$$
1=\frac{T V_{0}}{2} \sum_{\boldsymbol{k}, n}\left[1-\frac{V_{x}^{2}}{\lambda(\boldsymbol{k})}\right] \frac{1}{\omega_{n}^{2}+\chi_{-}^{2}(\boldsymbol{k})}+\left[1+\frac{V_{x}^{2}}{\lambda(\boldsymbol{k})}\right] \frac{1}{\omega_{n}^{2}+\chi_{+}(\boldsymbol{k})^{2}},
$$

where $\chi_{ \pm}(\boldsymbol{k})=\left[V_{x}^{2}+\beta^{2}(\boldsymbol{k})+\Delta^{2}+\xi^{2}(\boldsymbol{k}) \pm 2 \lambda(\boldsymbol{k})\right]^{1 / 2}, \lambda(\boldsymbol{k})=\left\{V_{x}^{2}\left[\Delta^{2}+\xi^{2}(\boldsymbol{k})\right]+\beta^{2}(\boldsymbol{k}) \xi^{2}(\boldsymbol{k})\right\}^{1 / 2}$. Then we sum over the Matsubara frequencies

$$
1=\frac{V_{0}}{2} \sum_{k}\left[1-\frac{V_{x}^{2}}{\lambda(k)}\right] \frac{1}{2 \chi_{-}(k)} \tanh \left[\frac{\chi_{-}(k)}{2 T}\right]+\left[1+\frac{V_{x}^{2}}{\lambda(k)}\right] \frac{1}{2 \chi_{+}(k)} \tanh \left[\frac{\chi_{+}(k)}{2 T}\right] .
$$

By replacing $\sum_{k}$ with $\int_{-\Lambda}^{\Lambda} d \xi \mathcal{N}\left(\mu_{F}\right)(\Lambda$ : cutoff energy), the self-consistent equation becomes

$$
\begin{aligned}
\eta= & \int_{0}^{\sinh (\eta) / \delta} d x\left\{\left[1-\frac{v^{2}}{\sqrt{v^{2} \delta^{2}\left(1+x^{2}\right)+b^{2} \delta^{2} x^{2}}}\right] \frac{\delta}{2 \sqrt{v^{2}+b^{2}+\delta^{2}\left(1+x^{2}\right)-2 \sqrt{v^{2} \delta^{2}\left(1+x^{2}\right)+b^{2} \delta^{2} x^{2}}}}\right] \\
& \times \tanh \left[\frac{1.764 \sqrt{v^{2}+b^{2}+\delta^{2}\left(1+x^{2}\right)-2 \sqrt{v^{2} \delta^{2}\left(1+x^{2}\right)+b^{2} \delta^{2} x^{2}}}}{2 \tau}\right] \\
& \left.\left.+\left[1+\frac{v^{2}}{\sqrt{v^{2} \delta^{2}\left(1+x^{2}\right)+b^{2} \delta^{2} x^{2}}}\right] \frac{\delta}{2 \sqrt{v^{2}+b^{2}+\delta^{2}\left(1+x^{2}\right)+2 \sqrt{v^{2} \delta^{2}\left(1+x^{2}\right)+b^{2} \delta^{2} x^{2}}}}\right]\right\}, \\
& \left.\times \tanh \left[\frac{1.764 \sqrt{v^{2}+b^{2}+\delta^{2}\left(1+x^{2}\right)+2 \sqrt{v^{2} \delta^{2}\left(1+x^{2}\right)+b^{2} \delta^{2} x^{2}}}}{2 \tau}\right]\right\}
\end{aligned}
$$

where $\eta=\frac{1}{V_{0} \mathcal{N}\left(\mu_{F}\right)}, \quad b=\beta_{F} / \Delta_{0}, \quad \delta=\Delta\left(V_{x}\right) / \Delta_{0}$, $v=V_{x} / \Delta_{0}, \tau=T / T_{c}, x=\xi / \Delta\left(V_{x}\right) . \mathcal{N}\left(\mu_{F}\right)$ and $\beta_{F}$ are the density of states and the spin-orbit coupling strength near Fermi energy, $\Delta_{0}=1.764 T_{c}$ is the pairing gap at zero temperature without magnetic field, with $T_{c}$ being the critical temperature. Note that the effect of SOCs on $T_{c}$ is negligible [34].

Also note that the gap equation only gives the saddle point of the free energy. To determine the pairing gap and the phase transition point, one needs to further compare the superconducting free energy $\mathcal{F}_{s}$ and the normal state free energy $\mathcal{F}_{n}$. One way is to work out $\mathcal{F}_{s}-\mathcal{F}_{n}$ and evaluate $\int_{0}^{\Delta} d \Delta \frac{d\left(1 / V_{0}\right)}{d \Delta} \Delta^{2}$ as done in Ref. [35], where the interacting strength $V_{0}$ and pairing gap $\Delta$ are given by the self-consistent gap equation.

However, because of the presence of both SOC and magnetic field, the gap equation gets too involved to be solved exactly. Instead, we make an estimation by including the leading-order magnetization energy and condensation energy in $\mathcal{F}_{s}$ and $\mathcal{F}_{n}$, which leads us to the following form: $F_{s}-$ $F_{n} \approx-\frac{1}{2} \chi_{s} B^{2}-\frac{1}{2} N\left(\mu_{F}\right) \Delta^{2}+\frac{1}{2} \chi_{n} B^{2}$, where $\chi_{s}, \chi_{n}$ are the superconducting and normal spin susceptibility and $B$ is the in-plane magnetic field. In this way, the upper critical field $B_{c 2}$ can be estimated as $u_{B} B_{c 2} \approx \frac{\Delta}{\sqrt{2} \sqrt{1-\chi_{s} / \chi_{n}}}$ [26]. For the Ising superconductor, the superconducting spin susceptibility is $\chi_{s}=\chi_{n}\left[1-\pi k_{B} T \sum_{n} \frac{1}{\omega_{n}^{2}+\Delta^{2}+\beta^{2}\left(\mu_{F}\right)} \frac{\Delta^{2}}{\sqrt{\omega_{n}^{2}+\Delta^{2}}}\right]$ according to Ref. [26]. Combining with the self-consistent gap equation, we can obtain $\chi_{s} / \chi_{n}$ at zero temperature as shown in Fig. 1(a). We see that $\chi_{s} / \chi_{n}$ increases with the Ising SOC strength and approaches 1 . This explains the enhancement of in-plane $B_{c 2}$ shown in Fig. 1(b).

\section{APPENDIX C: THE LOW-ENERGY EFFECTIVE HAMILTONIAN OF NANOWIRES ON ISING SUPERCONDUCTORS}

The Green's function of a bulk Ising superconductor is

$$
\mathcal{G}(\boldsymbol{k}, i \omega)=\left(\begin{array}{cc}
G(\boldsymbol{k}, \mathbf{0}, i \omega) & -F(\boldsymbol{k}, \mathbf{0}, i \omega) \\
-F^{\dagger}(\boldsymbol{k}, \mathbf{0}, i \omega) & -G^{T}(-\boldsymbol{k}, \mathbf{0},-i \omega)
\end{array}\right) .
$$

Here we parametrize $G(\boldsymbol{k}, i \omega)$ which can be obtained from the Gor'kov equations as $G_{+}(\boldsymbol{k}, i \omega)+G_{-}(\boldsymbol{k}, i \omega) \hat{\boldsymbol{g}}(\boldsymbol{k}) \cdot \boldsymbol{\sigma}$. In addition,

$$
\begin{aligned}
& G_{+}(\xi(\boldsymbol{k}), i \omega)=-\frac{1}{2}\left[\frac{i \omega+\xi_{+}(\boldsymbol{k})}{\varphi_{+}(\boldsymbol{k}, \mathbf{0}, \Delta, \omega)}+\frac{i \omega+\xi_{-}(\boldsymbol{k})}{\varphi_{-}(\boldsymbol{k}, \mathbf{0}, \Delta, \omega)}\right] \\
& G_{-}(\xi(\boldsymbol{k}), i \omega)=-\frac{1}{2}\left[\frac{i \omega+\xi_{+}(\boldsymbol{k})}{\varphi_{+}(\boldsymbol{k}, \mathbf{0}, \Delta, \omega)}-\frac{i \omega+\xi_{-}(\boldsymbol{k})}{\varphi_{-}(\boldsymbol{k}, \mathbf{0}, \Delta, \omega)}\right] .
\end{aligned}
$$

By integrating out the superconducting background, the Green's function of the nanaowire is

$$
G_{w}^{-1}\left(k_{x}, i \omega\right)=i \omega-H_{w}\left(k_{x}\right)-\Sigma\left(k_{x} ; i \omega\right) .
$$

Here, $\Sigma\left(k_{x} ; i \omega\right)=\int \frac{d \boldsymbol{k}_{\perp}}{(2 \pi)^{d-1}} \mathcal{T} \dagger \mathcal{G}(\boldsymbol{k}, i \omega) \mathcal{T}$ is the self-energy of the Ising superconductor. Then we expand $G_{w}^{-1}\left(k_{x}, i \omega\right)$ in the linear order of $\omega$ such that $G_{w}^{-1}\left(k_{x}, \omega\right)=\tilde{\mathcal{Z}}_{0}\left[i \omega-\hat{H}_{\mathrm{eff}}\left(k_{x}\right)\right]$. 
The low-energy effective Hamiltonian is thus given by

$$
\hat{H}_{\text {eff }}\left(k_{x}\right)=\left(\begin{array}{cc}
\tilde{h}\left(k_{x}\right) & \tilde{\Delta}\left(k_{x}\right) \\
\tilde{\Delta}\left(k_{x}\right)^{\dagger} & -\tilde{h}^{T}\left(-k_{x}\right)
\end{array}\right) .
$$

Here the normal part, with the wire Hamiltonian $h_{w}\left(k_{x}\right)=$ $\frac{\hbar^{2} k_{x}^{2}}{2 m}-\mu_{w}+\alpha_{R} k_{x} \sigma_{y}+V_{x} \sigma_{x}$, is given by

$$
\begin{aligned}
\tilde{h}\left(k_{x}\right)= & \frac{h_{w}\left(k_{x}\right)}{\tilde{\mathcal{Z}}_{0}}-\frac{\Gamma_{c}^{2}}{2 \tilde{\mathcal{Z}}_{0}} \int \frac{d \boldsymbol{k}_{\perp}}{2 \pi}\left[\frac{\xi_{+}(\boldsymbol{k})}{\xi_{+}^{2}(\boldsymbol{k})+\Delta^{2}}+\frac{\xi_{-}(\boldsymbol{k})}{\xi_{-}^{2}(\boldsymbol{k})+\Delta^{2}}\right] \\
& -\frac{\Gamma_{c}^{2}}{2 \tilde{\mathcal{Z}}_{0}} \int \frac{d \boldsymbol{k}_{\perp}}{2 \pi}\left[\frac{\xi_{+}(\boldsymbol{k})}{\xi_{+}^{2}(\boldsymbol{k})+\Delta^{2}}-\frac{\xi_{-}(\boldsymbol{k})}{\xi_{-}^{2}(\boldsymbol{k})+\Delta^{2}}\right] \hat{\boldsymbol{g}}(\boldsymbol{k}) \cdot \boldsymbol{\sigma} .
\end{aligned}
$$

The induced pairing gap function is $\tilde{\Delta}\left(k_{x}\right)=\left[\psi\left(k_{x}\right)+\boldsymbol{d}\left(k_{x}\right)\right.$. $\sigma] i \sigma_{y}$, where

$$
\begin{aligned}
\psi\left(k_{x}\right) & =-\frac{\Delta \Gamma_{c}^{2}}{\tilde{\mathcal{Z}}_{0}} \int \frac{d \boldsymbol{k}_{\perp}}{2 \pi} F_{s}(\boldsymbol{k}, i \omega) \\
& =-\frac{\Delta \Gamma_{c}^{2}}{2 \tilde{\mathcal{Z}}_{0}} \int \frac{d \boldsymbol{k}_{\perp}}{2 \pi}\left[\frac{1}{\xi_{+}^{2}(\boldsymbol{k})+\Delta^{2}}+\frac{1}{\xi_{-}^{2}(\boldsymbol{k})+\Delta^{2}}\right], \\
\boldsymbol{d}\left(k_{x}\right) & =-\frac{\Delta \Gamma_{c}^{2}}{\tilde{\mathcal{Z}}_{0}} \int \frac{d \boldsymbol{k}_{\perp}}{2 \pi} \boldsymbol{F}_{t}(\boldsymbol{k}, i \omega) \\
& =-\frac{\Delta \Gamma_{c}^{2}}{2 \tilde{\mathcal{Z}}_{0}} \int \frac{d \boldsymbol{k}_{\perp}}{2 \pi}\left[\frac{1}{\xi_{+}^{2}(\boldsymbol{k})+\Delta^{2}}-\frac{1}{\xi_{-}^{2}(\boldsymbol{k})+\Delta^{2}}\right] \hat{\boldsymbol{g}}(\boldsymbol{k}), \\
\tilde{\mathcal{Z}}_{0} & =1+\frac{\Gamma_{c}^{2}}{2} \int \frac{d \boldsymbol{k}_{\perp}}{2 \pi}\left[\frac{1}{\xi_{+}^{2}(\boldsymbol{k})+\Delta^{2}}+\frac{1}{\xi_{-}^{2}(\boldsymbol{k})+\Delta^{2}}\right] .
\end{aligned}
$$

For an Ising superconductor, we have $\boldsymbol{g}(\boldsymbol{k})=\beta(\boldsymbol{k}) \hat{\boldsymbol{e}}_{z}$. The induced SOC in Eq. (C6) and $\boldsymbol{d}$-vector in Eq. (C7) can be written as

$$
\begin{aligned}
& \beta_{I}\left(k_{x}\right)=\frac{\Gamma_{c}^{2}}{2 \tilde{\mathcal{Z}}_{0}} \int \frac{d \boldsymbol{k}_{\perp}}{2 \pi}\left[\frac{-\xi_{+}(\boldsymbol{k})}{\xi_{+}^{2}(\boldsymbol{k})+\Delta^{2}}+\frac{\xi_{-}(\boldsymbol{k})}{\xi_{-}^{2}(\boldsymbol{k})+\Delta^{2}}\right], \\
& \boldsymbol{d}\left(k_{x}\right)=\frac{\Delta \Gamma_{c}^{2}}{2 \tilde{\mathcal{Z}}_{0}} \int \frac{d \boldsymbol{k}_{\perp}}{2 \pi}\left[\frac{1}{\xi_{+}^{2}(\boldsymbol{k})+\Delta^{2}}-\frac{1}{\xi_{-}^{2}(\boldsymbol{k})+\Delta^{2}}\right] \hat{\boldsymbol{e}}_{z} .
\end{aligned}
$$

The above results can be further simplified by rewriting

$$
\begin{aligned}
F\left(k_{x}\right) & =\int \frac{d \boldsymbol{k}_{\perp}}{2 \pi} \frac{f\left(\boldsymbol{k}_{\perp}, k_{x}\right)}{\xi_{ \pm}^{2}\left(\boldsymbol{k}_{\perp}, k_{x}\right)+\Delta^{2}} \\
& \approx \frac{\pi}{\Delta}\left\langle\rho_{ \pm}\left(\boldsymbol{k}_{\perp}\right) f\left(\boldsymbol{k}_{\perp}, k_{x}\right)\right\rangle_{\left|\boldsymbol{k}_{\perp}\right|^{2}=k_{F}^{2}-k_{x}^{2}},
\end{aligned}
$$

where the density of states in a unit length of $\boldsymbol{k}_{\perp}$ is $\rho_{ \pm}\left(\boldsymbol{k}_{\perp}\right)=$ $\left|\nabla_{\boldsymbol{k}_{\perp}} \xi_{ \pm}(\boldsymbol{k})\right|^{-1}$, the cutoff energy $\Lambda \gg \Delta$, and $\langle\cdots\rangle_{\left|\boldsymbol{k}_{\perp}\right|^{2}=k_{F}^{2}-k_{x}^{2}}$

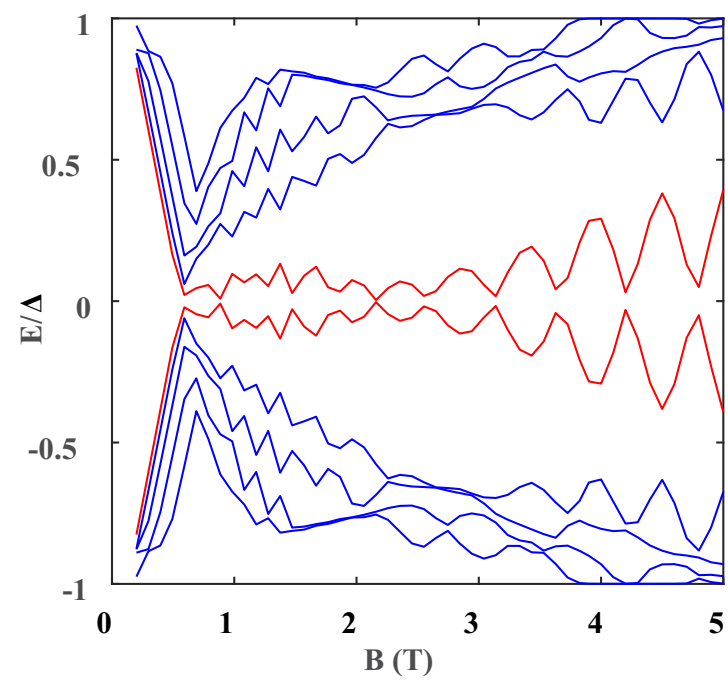

FIG. 5. Excitation energies of a finitely long nanowire on top of an Ising superconductor as a function of external magnetic field $B$. The lowest-lying fermionic states due to hybridized Majorana modes at two ends of the wire are highlighted in red.

denotes the average over the Fermi circles. The final results are

$$
\begin{aligned}
\psi\left(k_{x}\right) & =\frac{-\pi \Delta \Gamma_{c}^{2} \sum_{\lambda= \pm}\left\langle\rho_{\lambda}(\boldsymbol{k})\right\rangle_{\left|\boldsymbol{k}_{\perp}\right|^{2}=k_{F}^{2}-k_{x}^{2}}}{2 \Delta+\pi \Gamma_{c}^{2} \sum_{\lambda= \pm}\left\langle\rho_{\lambda}(\boldsymbol{k})\right\rangle_{\left|\boldsymbol{k}_{\perp}\right|^{2}=k_{F}^{2}-k_{x}^{2}}} \\
\boldsymbol{d}\left(k_{x}\right) & =\frac{-\pi \Delta \Gamma_{c}^{2}\left\langle\rho_{+}(\boldsymbol{k})-\rho_{-}(\boldsymbol{k})\right\rangle_{\left|\boldsymbol{k}_{\perp}\right|^{2}=k_{F}^{2}-k_{x}^{2}}}{2 \Delta+\pi \Gamma_{c}^{2} \sum_{\lambda= \pm}\left\langle\rho_{\lambda}(\boldsymbol{k})\right\rangle_{\left|\boldsymbol{k}_{\perp}\right|^{2}=k_{F}^{2}-k_{x}^{2}}} .
\end{aligned}
$$

The $D_{3 h}$ point group of a monolayer $2 \mathrm{H}-\mathrm{NbSe}_{2}$ dictates the SOC of the form $\beta(\boldsymbol{k})=\beta_{\mathrm{so}}\left[\sin \left(k_{x}\right)-\right.$ $2 \sin \left(k_{x} / 2\right) \cos \left(\sqrt{3} / 2 k_{y}\right)$ ] [36]. Therefore, near the $\Gamma$ point where the topological phase transition happens, $\psi\left(k_{x}\right) \approx \Delta_{0}, d_{z}\left(k_{x}\right) \propto k_{x} ;$ namely, $\psi\left(k_{x}\right)$ reduces to an $s$-wave-like gap, but $d_{z}\left(k_{x}\right)$ becomes a $p$-wave-like gap.

\section{APPENDIX D: ENERGY SPECTRUM OF A FINITELY LONG NANOWIRE ON TOP OF AN ISING SUPERCONDUCTOR}

To further confirm the existence of Majorana zero modes in Fig. 4, we calculate the energy spectrum of a finitely long wire on top of an Ising superconductor (Fig. 5). The excitation energy of the lowest-lying fermionic mode shows a clear oscillation as a function of external magnetic fields. This oscillatory behavior in the splitting energy is a characteristic of hybridized Majorana zero modes localized at the two ends of the finitely long wire.
[1] D. A. Ivanov, Phys. Rev. Lett. 86, 268 (2001).

[2] C. Nayak, S. H. Simon, A. Stern, M. Freedman, and S. Das Sarma, Rev. Mod. Phys. 80, 1083 (2008).

[3] N. Read and D. Green, Phys. Rev. B 61, 10267 (2000).
[4] A. Y. Kitaev, Phys. Usp. 44, 131 (2001).

[5] L. Fu and C. L. Kane, Phys. Rev. Lett. 100, 096407 (2008).

[6] Y. Oreg, G. Refael, and F. von Oppen, Phys. Rev. Lett. 105, 177002 (2010). 
[7] J. D. Sau, R. M. Lutchyn, S. Tewari, and S. Das Sarma, Phys. Rev. Lett. 104, 040502 (2010).

[8] T.-P. Choy, J. M. Edge, A. R. Akhmerov, and C. W. J. Beenakker, Phys. Rev. B 84, 195442 (2011).

[9] J. Klinovaja, P. Stano, A. Yazdani, and D. Loss, Phys. Rev. Lett. 111, 186805 (2013).

[10] S. Nadj-Perge, I. K. Drozdov, J. Li, H. Chen, S. Jeon, J. Seo, A. H. MacDonald, B. A. Bernevig, and A. Yazdani, Science 346, 602 (2014)

[11] C.-Z. Chen, Y.-M. Xie, J. Liu, P. A. Lee, and K. T. Law, Phys. Rev. B 97, 104504 (2018).

[12] V. Mourik, K. Zuo, S. M. Frolov, S. R. Plissard, E. P. A. M. Bakkers, and L. P. Kouwenhoven, Science 336, 1003 (2012).

[13] S. M. Albrecht, A. Higginbotham, M. Madsen, F. Kuemmeth, T. S. Jespersen, J. Nygård, P. Krogstrup, and C. Marcus, Nature (London) 531, 206 (2016).

[14] M. T. Deng, S. Vaitiekenas, E. B. Hansen, J. Danon, M. Leijnse, K. Flensberg, J. Nygård, P. Krogstrup, and C. M. Marcus, Science 354, 1557 (2016).

[15] H.-H. Sun, K.-W. Zhang, L.-H. Hu, C. Li, G.-Y. Wang, H.-Y. Ma, Z.-A. Xu, C.-L. Gao, D.-D. Guan, Y.-Y. Li, C. Liu, D. Qian, Y. Zhou, L. Fu, S.-C. Li, F.-C. Zhang, and J.-F. Jia, Phys. Rev. Lett. 116, 257003 (2016).

[16] H. Zhang, C.-X. Liu, S. Gazibegovic, D. Xu, J. A. Logan, G. Wang, N. van Loo, J. D. S. Bommer, M. W. A. de Moor, D. Car, R. L. M. Op het Veld, P. J. van Veldhoven, S. Koelling, M. A. Verheijen, M. Pendharkar, D. J. Pennachio, B. Shojaei, J. S. Lee, C. J. Palmstrøm, E. P. A. M. Bakkers, S. D. Sarma, and L. P. Kouwenhoven, Nature (London) 556, 74 (2018).

[17] D. Aasen, M. Hell, R. V. Mishmash, A. Higginbotham, J. Danon, M. Leijnse, T. S. Jespersen, J. A. Folk, C. M. Marcus, K. Flensberg, and J. Alicea, Phys. Rev. X 6, 031016 (2016).

[18] R. M. Lutchyn, E. P. A. M. Bakkers, L. P. Kouwenhoven, P. Krogstrup, C. M. Marcus, and Y. Oreg, Nat. Rev. Mater. 3, 52 (2018).

[19] H. Zhang, Ö. Gül, S. Conesa-Boj, M. P. Nowak, M. Wimmer, K. Zuo, V. Mourik, F. K. de Vries, J. van Veen, M. W. A. de Moor,
J. D. S. Bommer, D. J. van Woerkom, D. Car, S. R. Plissard, E. P. A. M. Bakkers, M. Quintero-Pérez, M. C. Cassidy, S. Koelling, S. Goswami, K. Watanabe, T. Taniguchi, and L. P. Kouwenhoven, Nat. Commun. 8, 16025 (2017).

[20] J. M. Lu, O. Zheliuk, I. Leermakers, N. F. Q. Yuan, U. Zeitler, K. T. Law, and J. T. Ye, Science 350, 1353 (2015).

[21] Y. Saito, Y. Nakamura, M. S. Bahramy, Y. Kohama, J. Ye, Y. Kasahara, Y. Nakagawa, M. Onga, M. Tokunaga, T. Nojima, Y. Yanase, and Y. Iwasa, Nat. Phys. 12, 144 (2015).

[22] X. Xi, Z. Wang, W. Zhao, J.-H. Park, K. T. Law, H. Berger, L. Forró, J. Shan, and K. F. Mak, Nat. Phys. 12, 139 (2015).

[23] B. T. Zhou, N. F. Q. Yuan, H.-L. Jiang, and K. T. Law, Phys. Rev. B 93, 180501(R) (2016).

[24] G. Sharma and S. Tewari, Phys. Rev. B 94, 094515 (2016).

[25] J. Zhang and V. Aji, Phys. Rev. B 94, 060501(R) (2016).

[26] P. A. Frigeri, D. F. Agterberg, and M. Sigrist, New J. Phys. 6, 115 (2004)

[27] L. P. Gor'kov and E. I. Rashba, Phys. Rev. Lett. 87, 037004 (2001).

[28] A. P. Mackenzie and Y. Maeno, Rev. Mod. Phys. 75, 657 (2003).

[29] E. Sohn, X. Xi, W.-Y. He, S. Jiang, Z. Wang, K. Kang, J.-H. Park, H. Berger, L. Forró, K. T. Law, J. Shan, and K. F. Mak, Nat. Mater. 17, 504 (2018).

[30] J. Alicea, Phys. Rev. B 81, 125318 (2010).

[31] G.-B. Liu, W.-Y. Shan, Y. Yao, W. Yao, and D. Xiao, Phys. Rev. B 88, 085433 (2013).

[32] W.-Y. He, B. T. Zhou, J. J. He, N. F. Q. Yuan, T. Zhang, and K. T. Law, Commun. Phys. 1, 40 (2018).

[33] C.-X. Liu, J. D. Sau, T. D. Stanescu, and S. Das Sarma, Phys. Rev. B 96, 075161 (2017).

[34] P. A. Frigeri, D. F. Agterberg, A. Koga, and M. Sigrist, Phys. Rev. Lett. 92, 097001 (2004).

[35] K. Maki and T. Tsuneto, Prog. Theor. Phys. 31, 945 (1964).

[36] E. Bauer and M. Sigrist, Non-Centrosymmetric Superconductors: Introduction and Overview (Springer-Verlag, Berlin, 2012). 